\title{
Twelve-month growth and accession of tolerance in infants with cow's milk protein allergy compared among those fed with breast milk or alternative formulae
}

\author{
Trakulpark C, Densupsoontorn N
}

\begin{abstract}
Background: Cow's milk protein allergy (CMPA) is a common food allergy in children. The impact of various feeding regimens on growth in infants with CMPA is not sufficiently well understood.
\end{abstract}

Objective: To investigate 12-month growth and accession of tolerance in infants with CMPA compared among those fed with breast milk or alternative formulae.

Methods: This retrospective study included CMPA infants with treatment adherence for at least six months. Infants were categorized into the following feeding regimen groups: soy-based formula (SF), extensively hydrolyzed protein formula (EHF), commercial amino acid-based formula (cAAF), new amino acid-based formula (nAAF), chicken-based formula, and breast milk. Weight-for-age z-score (WAZ), length-for-age z-score (LAZ), and weight-for-length z-score (WLZ) were evaluated at diagnosis and at follow-ups. Clinical manifestations, other allergenic foods, and time to tolerance of CMP were assessed.

Results: One hundred and sixteen infants were enrolled. Infants consuming EHF had markedly improved WAZ. Infants with one symptom at diagnosis, those who had gastrointestinal symptom, and those with allergy to only CMP had more pronounced growth improvement. Compared to breast milk, SF and EHF were significantly associated with lower probability of tolerance to CMP (HR: 0.14, 95\%CI: 0.03-0.62; and, HR: 0.21, 95\%CI: 0.07-0.64, respectively). Those allergic to only CMP were more likely to develop tolerance to CMP than those allergic to CMP and other foods.

Conclusion: Improvement in growth was significantly more pronounced in CMP-allergic infants fed with EHF. Accession of tolerance to CMP was associated with breast milk as the therapeutic diet.

Key words: Twelve-month growth, accession of tolerance, infants, cow's milk protein allergy, breast milk, alternative formulae

From:

Division of Nutrition, Department of Pediatrics

Faculty of Medicine Siriraj Hospital, Mahidol University, Bangkok, Thailand

\section{Introduction}

Cow's milk protein allergy (CMPA) is a common food allergy in children. Its incidence is approximately $2-3 \%$ among all infants, and $0.5 \%$ among breastfed infants. ${ }^{1}$ Clinical manifestation of CMPA usually presents before six months of age, but it can be later depending on the age of exposure to cow's milk protein (CMP). The presenting symptoms of CMPA can be found in one or more organ systems, including
Corresponding author:

Narumon Densupsoontorn

Division of Nutrition, Department of Pediatrics

Faculty of Medicine Siriraj Hospital, Mahidol University

2 Wanglang Road, Bangkoknoi, Bangkok 10700, Thailand

E-mail: narumon.den@mahidol.ac.th

gastrointestinal, cutaneous, and/or respiratory systems, or the symptom can be systemic in the form of anaphylaxis. ${ }^{2}$

The principles of CMPA treatment include avoidance of CMP and the provision of substitute formulae and replacement foods that are age-appropriate and nutrient-rich to promote normal growth. Maternal CMP avoidance is essential for breast-fed infants, whereas a hypoallergenic formula is used as 
an alternative therapy in formula-fed infants. Generally, commercial hypoallergenic formulae include amino acid-based formula (AAF) and extensively hydrolyzed protein formula (EHF). However, soy protein-based formula (SF) may be considered in infants older than six months, especially among those with immunoglobulin E ( $\operatorname{IgE}$ )-mediated CMPA due to the low prevalence of concomitant allergy to soy protein. $.^{3-5} \mathrm{Ji}-$ rapinyo et $\mathrm{al}^{6-9}$ studied the efficacy of two innovative alternative formulae, including a chicken-based formula $(\mathrm{CF})$ and a new amino acid-based formula (nAAF), both of which were found to be well-tolerated in infants with CMPA. Briefly, the components of the chicken-based formula include homogenized chicken meat, glucose polymers from corn starch, vegetable oils, and micronutrients; and, the components of nAAF include amino acids, glucose polymers from rice starch, vegetable oils, and micronutrients. Protein derived from rice glucose polymers in nAAF was detected in the amount of 0.04 $\mathrm{g} \%$ of nitrogen content using the Kjeldahl method in contrast to that obtained from corn glucose polymers in cAAF, which was $0.2 \mathrm{~g} \%$ of nitrogen content. ${ }^{8}$

Children with food allergies are at risk for nutrient deficiencies and retarded growth due to unnecessary food avoidance. Food allergies have a negative effect on linear growth. ${ }^{10-14}$ Moreover, children who are allergic to multiple foods are shorter than those with a single food allergy. ${ }^{13}$

Studies of growth in children with CMPA treated with medical formulae have been reported. Children with IgE-mediated CMPA treated with a hydrolyzed rice protein formula demonstrated no difference in weight or length compared to those who received an extensively hydrolyzed whey (EHWF) and casein formula, and their growth parameters up to two years of age were within normal range. ${ }^{15}$ Infants who received an extensively hydrolyzed casein-based formula (EHCF) for four months had significantly increased weight-for-age $\mathrm{z}$-score (WAZ), length-for-age $\mathrm{z}$-score (LAZ), and weight-for-length Z-score (WLZ). ${ }^{16}$ Growth of children with CMPA treated with the chicken-based formula was reported to be the same as the growth of normal children. ${ }^{17}$ Infants who did not respond to EHF had a significant increase in weight $\mathrm{z}$-score change after 12 weeks of consuming AAF. ${ }^{18}$

While growth and diet tolerance of CMPA infants may have been previously studied, growth and tolerance of Thai CMPA infants compared among those receiving various feeding regimens has not been widely studied, except for those treated with chicken-based formula. Also, we were namely focusing in this study on such aspects in infants fed with two alternative, innovative formulas developed by Thai researchers, i.e. the chicken-based formula and the new amino acid-based formula. Accordingly, this study was aimed to investigate 12-month growth and accession of tolerance in infants with CMPA compared among those fed with breast milk or alternative formulae. Time to and the factors associated with development of tolerance to CMP were also evaluated.

\section{Methods \\ Subjects}

The protocol was approved by the Siriraj Institutional Review Board of the Faculty of Medicine Siriraj Hospital, Mahidol University, Bangkok, Thailand (COA number:
Si 369/2019). This retrospective study reviewed data of infants diagnosed with CMPA at the Pediatric Nutrition Clinic, Pediatric Allergy Clinic, and Pediatric Gastrointestinal Clinic of the Department of Pediatrics, Faculty of Medicine Siriraj Hospital during January 2008 to April 2019. Diagnosis of CMPA in this study was established based on the disappearance of symptoms after food elimination, and relapse upon open oral food re-challenge following resolution of symptoms after food elimination. IgE-mediated CMPA was documented by higher levels of serum specific immunoglobulin E (sIgE) or positive skin prick test.

Infants aged 1 month to 12 months who were diagnosed with CMPA and who adhered to the prescribed treatment for at least 6 months were eligible for inclusion. Alternative formulae consisted of SF, EHF, AAF (including commercial amino acid-based formula [cAAF] and nAAF), and chicken-based formula. Breast milk along with maternal CMP avoidance was the therapeutic regimen for breast-fed infants.

Infants having one or both of the following conditions were excluded: (i) coexisting chronic diseases that might affect normal growth, such as preterm status, congenital heart disease, chronic lung disease, chronic diarrhea, chronic kidney disease, metabolic disease, or genetic disorders; and/or, (ii) regular consumption of more than one type of formulae or milk.

\section{Growth and clinical data}

Clinical allergic manifestations categorized by involved systems, initial blood eosinophil count, and serum levels of cow's milk-specific IgE were evaluated. Anthropometric data, including weight and length, were evaluated at diagnosis and at all follow-up visits (3, 6, 9, and 12 months after diagnosis). Weight-for-age z-score, LAZ, and WLZ were calculated based upon the World Health Organization (WHO) Child Growth Standards using the WHO Anthro Survey Analyser. ${ }^{19}$ A value of WAZ, LAZ, or WLZ between -2 and 2 indicated normal growth status. ${ }^{20}$ Blood eosinophil counts of more than 450 cells $/ \mathrm{mm}^{3}$ was defined as eosinophilia. The cut-off of serum cow's milk-specific IgE level was used to define as positive when it was $0.35 \mathrm{kUA} / \mathrm{L}$ or more. Data specific to other allergenic complementary foods were also analyzed. Tolerance to CMP was evaluated by open oral food challenge under the supervision of an attending pediatrician of one of the three clinics aforementioned. Time until the development of tolerance to CMP was also evaluated.

\section{Data analysis}

For baseline data, categorical variables were presented as number and percentage, and they were compared among groups using chi-square test or Fisher's exact test. Non-normally distributed continuous data were expressed as median (P25, P75), and they were compared among groups using Kruskal Wallis $\mathrm{H}$ test. Normally distributed continuous data were shown as mean \pm standard deviation (SD), and they were compared among groups using analysis of variance (ANOVA) test. Growth parameters were presented as mean of $\mathrm{z}$-score $\pm \mathrm{SD}$, and data between follow-up visits and baseline were compared using paired Student's $t$-test. Cox regression analysis was used to assess factors significantly associated with 
the development of tolerance to CMP. Kaplan-Meier survival analysis was used to analyze the median time to tolerance of CMP among feeding regimens, and differences in median time among feeding regimens were analyzed by log-rank test.

Univariate and multivariate Cox regression analyses were used to identify factors independently associated with the development of tolerance to CMPA, and those results are shown at hazard ratio (HR) and 95\% confidence interval (CI). The data were analyzed using SPSS Statistics version 18 (SPSS, Inc., Chicago, IL, USA). All tests of statistical significance were two-sided, and a $p$-value of less than 0.05 indicated statistical significance.

\section{Results}

Of the 198 infants that were recruited into this study, 68 and 14 infants were excluded due to the presence of coexisting chronic diseases and consumption of more than one type of alternative formulae or milk, respectively. The remaining 116 infants were included, and they had the following characteristics: 68 males, median age 4.1 months $(2.2,7.4)$, mean weight $6.3 \pm 1.8 \mathrm{~kg}$, mean WAZ $-0.76 \pm 1.21$, mean length $62.5 \pm 6.7 \mathrm{~cm}$, mean LAZ $-0.64 \pm 1.06$, and mean WLZ $-0.38 \pm 1.23$. Thirty-two infants $(27 \%)$ were allergic to foods other than CMP. Twelve infants (10\%) had another food allergy, and twenty infants (17\%) had more than one other food allergy. Twenty-one infants were allergic to egg white, and 17 were allergic to egg yolk. Twelve infants were allergic to legumes, including soybean $(n=11)$ and peanut $(n=1)$. Nine, nine, and one infants were allergic to seafood, wheat, and chicken, respectively.

Infants were categorized according to type of alternative formulae or milk, as follows: SF ( $n=21)$, EHF $(n=60)$, cAAF $(\mathrm{n}=11), \operatorname{nAAF}(\mathrm{n}=5)$, chicken-based formula $(\mathrm{n}=9)$, and breast milk $(n=10)$. The median age at diagnosis was significantly different among groups, as follows: SF 6.0 months, EHF 3.8 months, cAAF 5.5 months, nAAF 2.8 months, chicken-based formula 9.0 months, and breast milk 2.6 months ( $p$ $=0.001$ ) (Table 1). The other baseline demographic and clinical characteristics of six groups, including mean WAZ, LAZ, and WLZ, IgE-mediated CMPA type, presenting symptoms

Table 1. Baseline demographic and clinical characteristics of 116 infants compared among milk groups

\begin{tabular}{|c|c|c|c|c|c|c|c|}
\hline \multirow[b]{2}{*}{ Characteristics } & \multicolumn{7}{|c|}{ Milk groups } \\
\hline & $\begin{array}{c}\text { SF } \\
(n=21)\end{array}$ & $\begin{array}{c}\text { EHF } \\
(n=60)\end{array}$ & $\begin{array}{l}\mathrm{cAAF} \\
(\mathrm{n}=11)\end{array}$ & $\begin{array}{l}\text { nAAF } \\
(n=5)\end{array}$ & $\begin{array}{c}\text { CF } \\
(n=9)\end{array}$ & $\begin{array}{l}\text { Breast milk } \\
\quad(\mathrm{n}=10)\end{array}$ & $p$-value \\
\hline Male gender, n (\%) & $16(76)$ & $28(47)$ & $6(55)$ & $4(80)$ & $5(56)$ & $9(90)$ & 0.040 \\
\hline Age at diagnosis (months), median (P25, P75) & $6.0(3.7,8.3)$ & $3.8(2.1,6.0)$ & $5.5(2.2,10.5)$ & $2.8(1.9,6.7)$ & $9.0(4.8,10.4)$ & $2.6(1.1,3.8)$ & 0.001 \\
\hline \multicolumn{8}{|l|}{ Nutritional status z-score, mean \pm SD } \\
\hline Weight-for-age & $-0.31 \pm 1.24$ & $-0.74 \pm 1.11$ & $-1.30 \pm 1.63$ & $-1.77 \pm 1.49$ & $-0.66 \pm 0.94$ & $-0.77 \pm 0.98$ & 0.124 \\
\hline Length-for-age & $-0.39 \pm 0.96$ & $-0.60 \pm 1.01$ & $-1.05 \pm 1.36$ & $-1.79 \pm 1.31$ & $-0.56 \pm 1.00$ & $-0.49 \pm 0.97$ & 0.107 \\
\hline Weight-for-length & $-0.03 \pm 1.37$ & $-0.35 \pm 1.18$ & $-0.88 \pm 1.55$ & $-0.48 \pm 0.93$ & $-0.49 \pm 0.78$ & $-0.55 \pm 1.39$ & 0.593 \\
\hline IgE-mediated CMPA, n (\%) & $8(38)$ & $13(22)$ & $2(18)$ & $2(40)$ & $0(0)$ & $0(0)$ & 0.068 \\
\hline \multicolumn{8}{|l|}{ Presenting symptoms, $\mathrm{n}(\%)^{+}$} \\
\hline Gastrointestinal & $10(48)$ & $31(52)$ & $8(73)$ & $1(20)$ & $2(22)$ & $6(60)$ & 0.212 \\
\hline Cutaneous & $13(62)$ & $34(57)$ & $5(45)$ & $4(80)$ & $5(56)$ & $4(40)$ & 0.731 \\
\hline Respiratory & $3(14)$ & $5(8)$ & $2(18)$ & $0(0)$ & $5(56)$ & $1(10)$ & 0.020 \\
\hline Anaphylaxis & $0(0)$ & $0(0)$ & $0(0)$ & $0(0)$ & $0(0)$ & $0(0)$ & - \\
\hline Number of presenting symptoms, $\mathrm{n}(\%)$ & & & & & & & 0.319 \\
\hline 1 symptom & $16(76)$ & $51(85)$ & $7(64)$ & $5(100)$ & $6(67)$ & $9(90)$ & \\
\hline$>1$ symptom & $5(24)$ & $9(15)$ & $4(36)$ & $0(0)$ & $3(33)$ & $1(10)$ & \\
\hline Blood eosinophil, n (\%) & & & & & & & 0.608 \\
\hline$>450$ cells $/ \mathrm{mm}^{3}$ & $5(31)$ & $13(29)$ & $4(40)$ & $3(60)$ & $4(50)$ & $4(44)$ & \\
\hline$\leq 450$ cells $/ \mathrm{mm}^{3}$ & $11(69)$ & $32(71)$ & $6(60)$ & $2(40)$ & $4(50)$ & $5(56)$ & \\
\hline Other food allergies, n (\%) & $5(24)$ & $16(27)$ & $4(36)$ & $3(60)$ & $1(11)$ & $3(30)$ & 0.502 \\
\hline
\end{tabular}

Abbreviations: SF: soy protein-based formula; EHF: extensively hydrolyzed protein formula; cAAF: commercial amino acid-based formula; nAAF: new amino acid-based formula; CF: chicken-based formula; SD: standard deviation; IgE: immunoglobulin E; CMPA: cow's milk protein allergy

${ }^{\dagger}$ Each subject may have had more than one symptom.

${ }^{\circledR}$ Blood eosinophil count was analyzed only in subjects whose blood was measured.

A $p$-value $<0.05$ indicates statistical significance. 
and their number, eosinophil count, and the presence of other food allergies, were not significantly different among groups, except for gender and respiratory presenting symptom. Among infants fed with breast milk, allergic proctocolitis was diagnosed in 50\%, atopic dermatitis in $40 \%$, and food protein-induced enterocolitis in $10 \%$. Atopic dermatitis was diagnosed in $57 \%$ of infants treated with SF, in 55\% treated with EHF, in $45 \%$ treated with cAAF, in $80 \%$ treated with nAAF, and in $44 \%$ treated with CF. Food protein-induced enterocolitis was found in 5 infants, including 2 treated with EHF, 2 treated with $\mathrm{CAAF}$, and 1 treated with breast milk.

Some infants received nutrition supplements including multivitamins, ferrous sulfate, or zinc sulfate, as follows: SF (n $=3), \operatorname{EHF}(n=2), \operatorname{cAAF}(n=2), \operatorname{nAAF}(n=0), \operatorname{CF}(n=0)$, and breast milk $(n=2)$. These supplements were not significantly different among groups.

The weight and length of 103 infants (88\%) at 3 months, $95(81 \%)$ at 6 months, $92(79 \%)$ at 9 months, and $61(52 \%)$ at 12 months of follow-up were calculated into Z-scores (Table 2). An overall improvement in WAZ, LAZ, and WLZ was observed in most infants within the 12-month study. Infants consuming EHF had significantly increased WAZ throughout the 12-month study compared to baseline and those consuming SF had significantly increased WAZ at 12 months compared to baseline. Weight-for-age z-score of infants who consumed cAAF and nAAF tended to increase at 6 and 12 months, respectively, compared to baseline. Weight-for-age $\mathrm{z}$-score of infants who consumed either chicken-based formula or breast milk during 12 months did not change significantly compared to baseline. Length-for-age z-score improved significantly only in the EHF group at the 3-, 6-, and 9-month follow-ups, and in the nAAF group at the 9- and 12-month follow-ups. Weight-for-length $\mathrm{z}$-score improved significantly only in the EHF group at the 9- and 12-month follow-ups, and in the SF group at the 12-month follow-up. Regarding the remaining subjects at the 12-month follow-up visit, $48 \%$ were still in the study in the SF group, $57 \%$ in the EHF group, $45 \%$ in the cAAF group, $80 \%$ in the $\mathrm{nAAF}$ group, $33 \%$ in the $\mathrm{CF}$ group, and $50 \%$ in the breast milk group.

Table 2. Infant growth parameters at diagnosis and at each follow-up time point for each feeding regimen

\begin{tabular}{|c|c|c|c|c|c|}
\hline Feeding group & At diagnosis & $3^{\text {rd }}$ month & $6^{\text {th }}$ month & $9^{\text {th }}$ month & $12^{\text {th }}$ month \\
\hline \multicolumn{6}{|c|}{ Weight-for-age $z$-score, mean \pm SD } \\
\hline Soy formula & $-0.31 \pm 1.24$ & $-0.30 \pm 1.18$ & $-0.22 \pm 1.18$ & $0.01 \pm 1.28$ & $0.28 \pm 1.56$ \\
\hline $\mathrm{N}$ & 21 & 18 & 19 & 17 & 10 \\
\hline$p$-value vs. baseline & & 0.627 & 0.105 & 0.063 & 0.031 \\
\hline EHF & $-0.74 \pm 1.11$ & $-0.38 \pm 0.96$ & $-0.13 \pm 1.00$ & $-0.03 \pm 1.07$ & $0.14 \pm 1.16$ \\
\hline $\mathrm{N}$ & 60 & 57 & 47 & 48 & 34 \\
\hline$p$-value vs. baseline & & 0.001 & 0.000 & 0.000 & 0.000 \\
\hline cAAF & $-1.30 \pm 1.63$ & $-0.85 \pm 1.39$ & $-0.60 \pm 1.57$ & $-0.81 \pm 1.78$ & $-0.95 \pm 2.41$ \\
\hline $\mathrm{N}$ & 11 & 11 & 10 & 9 & 5 \\
\hline$p$-value vs. baseline & & 0.181 & 0.058 & 0.152 & 0.224 \\
\hline $\mathrm{nAAF}$ & $-1.77 \pm 1.49$ & $-1.53 \pm 0.80$ & $-1.21 \pm 0.86$ & $-0.50 \pm 0.73$ & $-0.22 \pm 0.78$ \\
\hline $\mathrm{N}$ & 5 & 3 & 3 & 4 & 4 \\
\hline$p$-value vs. baseline & & 0.536 & 0.186 & 0.112 & 0.062 \\
\hline Chicken-based formula & $-0.66 \pm 0.94$ & $-0.59 \pm 1.22$ & $-0.10 \pm 1.05$ & $-0.32 \pm 0.81$ & $-1.31 \pm 0.63$ \\
\hline $\mathrm{N}$ & 9 & 6 & 6 & 5 & 3 \\
\hline$p$-value vs. baseline & & 0.271 & 0.668 & 0.192 & 0.902 \\
\hline Breast milk & $-0.77 \pm 0.98$ & $-0.63 \pm 0.85$ & $-0.76 \pm 0.94$ & $-0.61 \pm 1.10$ & $-0.63 \pm 1.24$ \\
\hline $\mathrm{N}$ & 10 & 8 & 10 & 9 & 5 \\
\hline$p$-value vs. baseline & & 0.531 & 0.983 & 0.787 & 0.706 \\
\hline All groups & $-0.76 \pm 1.21$ & $-0.48 \pm 1.06$ & $-0.30 \pm 1.11$ & $-0.19 \pm 1.18$ & $-0.08 \pm 1.36$ \\
\hline $\mathrm{N}$ & 116 & 103 & 95 & 92 & 61 \\
\hline$p$-value vs. baseline & & 0.000 & 0.000 & 0.000 & 0.000 \\
\hline
\end{tabular}


Table 2. (continued)

\begin{tabular}{|c|c|c|c|c|c|}
\hline Feeding group & At diagnosis & $3^{\text {rd }}$ month & $6^{\text {th }}$ month & $9^{\text {th }}$ month & $12^{\text {th }}$ month \\
\hline \multicolumn{6}{|c|}{ Length-for-age $\mathrm{z}$-score, mean \pm SD } \\
\hline Soy formula & $-0.39 \pm 0.96$ & $-0.23 \pm 0.93$ & $-0.30 \pm 1.08$ & $-0.21 \pm 0.97$ & $-0.11 \pm 1.08$ \\
\hline$p$-value vs. baseline & & 0.070 & 0.440 & 0.323 & 0.593 \\
\hline EHF & $-0.60 \pm 1.01$ & $-0.28 \pm 0.92$ & $-0.27 \pm 0.96$ & $-0.25 \pm 1.02$ & $-0.17 \pm 0.99$ \\
\hline$p$-value vs. baseline & & 0.004 & 0.045 & 0.021 & 0.090 \\
\hline cAAF & $-1.05 \pm 1.36$ & $-1.04 \pm 1.66$ & $-0.63 \pm 1.87$ & $-0.88 \pm 1.70$ & $-1.21 \pm 2.06$ \\
\hline$p$-value vs. baseline & & 0.972 & 0.108 & 0.251 & 0.505 \\
\hline nAAF & $-1.79 \pm 1.31$ & $-1.20 \pm 1.48$ & $-1.24 \pm 1.09$ & $0.01 \pm 0.85$ & $-0.43 \pm 0.96$ \\
\hline$p$-value vs. baseline & & 0.226 & 0.288 & 0.030 & 0.018 \\
\hline Chicken-based formula & $-0.56 \pm 1.00$ & $-0.83 \pm 0.99$ & $-0.37 \pm 0.60$ & $-0.65 \pm 0.87$ & $-1.03 \pm 0.22$ \\
\hline$p$-value vs. baseline & & 0.968 & 0.523 & 0.742 & 0.974 \\
\hline Breast milk & $-0.49 \pm 0.97$ & $-0.74 \pm 1.40$ & $-0.57 \pm 0.80$ & $-0.47 \pm 0.78$ & $-0.49 \pm 1.21$ \\
\hline$p$-value vs. baseline & & 0.834 & 0.625 & 0.511 & 0.626 \\
\hline All groups & $-0.64 \pm 1.06$ & $-0.45 \pm 1.10$ & $-0.38 \pm 1.07$ & $-0.33 \pm 1.06$ & $-0.33 \pm 1.12$ \\
\hline$p$-value vs. baseline & & 0.002 & 0.010 & 0.001 & 0.013 \\
\hline \multicolumn{6}{|c|}{ Weight-for-length z-score, mean \pm SD } \\
\hline Soy formula & $-0.03 \pm 1.37$ & $-0.20 \pm 1.25$ & $-0.08 \pm 1.22$ & $0.14 \pm 1.29$ & $0.38 \pm 1.51$ \\
\hline$p$-value vs. baseline & & 0.407 & 0.540 & 0.342 & 0.030 \\
\hline EHF & $-0.35 \pm 1.18$ & $-0.22 \pm 1.08$ & $0.03 \pm 1.07$ & $0.11 \pm 1.09$ & $0.31 \pm 1.17$ \\
\hline$p$-value vs. baseline & & 0.212 & 0.101 & 0.007 & 0.005 \\
\hline cAAF & $-0.88 \pm 1.55$ & $-0.24 \pm 0.95$ & $-0.30 \pm 0.98$ & $-0.53 \pm 1.43$ & $-0.41 \pm 1.95$ \\
\hline$p$-value vs. baseline & & 0.123 & 0.139 & 0.255 & 0.328 \\
\hline nAAF & $-0.48 \pm 0.93$ & $-1.10 \pm 0.14$ & $-0.79 \pm 1.01$ & $-0.64 \pm 0.98$ & $-0.01 \pm 0.72$ \\
\hline$p$-value vs. baseline & & 0.577 & 0.434 & 0.366 & 0.117 \\
\hline Chicken-based formula & $-0.49 \pm 0.78$ & $-0.25 \pm 1.07$ & $0.09 \pm 1.20$ & $-0.02 \pm 0.86$ & $-1.11 \pm 0.86$ \\
\hline$p$-value vs. baseline & & 0.314 & 0.462 & 0.235 & 0.940 \\
\hline Breast milk & $-0.55 \pm 1.39$ & $-0.04 \pm 1.20$ & $-0.58 \pm 1.22$ & $-0.52 \pm 1.32$ & $-0.54 \pm 1.49$ \\
\hline$p$-value vs. baseline & & 0.639 & 0.936 & 0.652 & 0.965 \\
\hline All groups & $-0.38 \pm 1.23$ & $-0.23 \pm 1.08$ & $-0.10 \pm 1.11$ & $-0.04 \pm 1.17$ & $0.10 \pm 1.31$ \\
\hline$p$-value vs. baseline & & 0.127 & 0.029 & 0.005 & 0.001 \\
\hline
\end{tabular}

Abbreviations: SD: standard deviation; EHF: extensively hydrolyzed protein formula; cAAF: commercial amino acid-based formula; nAAF: new amino acidbased formula

A $p$-value $<0.05$ indicates statistical significance (difference between diagnosis and $3^{\text {rd }}, 6^{\text {th }}, 9^{\text {th }}$, and $12^{\text {th }}$ month; paired Student's $t$-test). 
Growth parameters categorized by isolated presenting symptom revealed improvement in the means of WAZ, LAZ, and WLZ in subjects who had either gastrointestinal or cutaneous symptom, whereas no significant change in growth was found in the respiratory group (Table 3). Moreover, infants with only one symptom at diagnosis had significantly increased WAZ, LAZ, and WLZ nearly throughout the 12 -month period relative to baseline, whereas those with more than one symptom showed improvement in WAZ and LAZ at the 6- and 9-month follow-ups (Table 3).
Infants who were allergic to only CMP had significant increases in the means of WAZ and LAZ throughout the 12-month period and in WLZ at the 9- and 12-month follow-ups. In contrast, those who were allergic to CMP and another food had increased WAZ only at the 12-month follow-up. Infants allergic to CMP and more than one other food had no significant improvement in growth parameters (Figure 1).

Table 3. Infant growth parameters at diagnosis and at each follow-up time point according to the presenting symptom

\begin{tabular}{|c|c|c|c|c|c|}
\hline $\begin{array}{c}\text { Body system associated with and number of the } \\
\text { presenting symptoms }\end{array}$ & At diagnosis & $3^{\text {rd }}$ month & $6^{\text {th }}$ month & $9^{\text {th }}$ month & $12^{\text {th }}$ month \\
\hline \multicolumn{6}{|l|}{ Weight-for-age $\mathrm{z}$-score, mean $\pm \mathrm{SD}$} \\
\hline \multicolumn{6}{|l|}{ Body system associated with the presenting symptoms } \\
\hline Cutaneous & $-0.37 \pm 0.85$ & $-0.32 \pm 0.87$ & $-0.23 \pm 0.84$ & $-0.08 \pm 0.95$ & $0.28 \pm 0.95$ \\
\hline $\mathrm{N}$ & 45 & 41 & 41 & 37 & 24 \\
\hline$p$-value vs. baseline & & 0.877 & 0.197 & 0.027 & 0.002 \\
\hline Gastrointestinal & $-1.32 \pm 1.47$ & $-0.80 \pm 1.18$ & $-0.57 \pm 1.43$ & $-0.35 \pm 1.48$ & $-0.29 \pm 1.73$ \\
\hline $\mathrm{N}$ & 41 & 35 & 30 & 34 & 23 \\
\hline$p$-value vs. baseline & & 0.000 & 0.001 & 0.000 & 0.001 \\
\hline Respiratory & $-0.42 \pm 1.18$ & $0.05 \pm 1.27$ & $-0.01 \pm 1.41$ & $-0.00 \pm 1.36$ & $-1.49 \pm 1.27$ \\
\hline $\mathrm{N}$ & 8 & 6 & 7 & 6 & 2 \\
\hline$p$-value vs. baseline & & 0.049 & 0.215 & 0.169 & 0.648 \\
\hline \multicolumn{6}{|l|}{ Number of the presenting symptoms } \\
\hline 1 symptom & $-0.79 \pm 1.26$ & $-0.50 \pm 1.06$ & $-0.34 \pm 1.15$ & $-0.19 \pm 1.23$ & $-0.05 \pm 1.41$ \\
\hline $\mathrm{N}$ & 94 & 82 & 78 & 77 & 49 \\
\hline$p$-value vs. baseline & & 0.000 & 0.000 & 0.000 & 0.000 \\
\hline$>1$ symptom & $-0.62 \pm 0.93$ & $-0.41 \pm 1.07$ & $-0.08 \pm 0.88$ & $-0.20 \pm 0.89$ & $-0.18 \pm 1.17$ \\
\hline $\mathrm{N}$ & 22 & 21 & 17 & 15 & 12 \\
\hline$p$-value vs. baseline & & 0.065 & 0.017 & 0.077 & 0.109 \\
\hline \multicolumn{6}{|l|}{ Length-for-age $\mathrm{z}$-score, mean $\pm \mathrm{SD}$} \\
\hline \multicolumn{6}{|l|}{ Body system associated with the presenting symptoms } \\
\hline Cutaneous & $-0.60 \pm 0.85$ & $-0.31 \pm 0.91$ & $-0.55 \pm 0.88$ & $-0.29 \pm 0.85$ & $-0.12 \pm 0.78$ \\
\hline$p$-value vs. baseline & & 0.019 & 0.582 & 0.062 & 0.031 \\
\hline Gastrointestinal & $-0.89 \pm 1.30$ & $-0.78 \pm 1.24$ & $-0.48 \pm 1.37$ & $-0.58 \pm 1.31$ & $-0.59 \pm 1.52$ \\
\hline$p$-value vs. baseline & & 0.164 & 0.040 & 0.034 & 0.162 \\
\hline Respiratory & $-0.38 \pm 0.61$ & $-0.34 \pm 0.76$ & $-0.37 \pm 0.50$ & $-0.50 \pm 0.62$ & $-1.34 \pm 0.39$ \\
\hline$p$-value vs. baseline & & 0.111 & 0.908 & 0.765 & 0.397 \\
\hline \multicolumn{6}{|l|}{ Number of the presenting symptoms } \\
\hline 1 symptom & $-0.71 \pm 1.06$ & $-0.51 \pm 1.07$ & $-0.51 \pm 1.06$ & $-0.43 \pm 1.06$ & $-0.39 \pm 1.20$ \\
\hline$p$-value vs. baseline & & 0.005 & 0.054 & 0.007 & 0.030 \\
\hline$>1$ symptom & $-0.35 \pm 1.05$ & $-0.18 \pm 1.19$ & $0.17 \pm 0.96$ & $0.16 \pm 0.91$ & $-0.09 \pm 0.71$ \\
\hline$p$-value vs. baseline & & 0.233 & 0.047 & 0.009 & 0.143 \\
\hline
\end{tabular}


Table 3. (Continued)

\begin{tabular}{|c|c|c|c|c|c|}
\hline $\begin{array}{l}\text { Body system associated with and number of the } \\
\text { presenting symptoms }\end{array}$ & At diagnosis & $3^{\text {rd }}$ month & $6^{\text {th }}$ month & $9^{\text {th }}$ month & $12^{\text {th }}$ month \\
\hline \multicolumn{6}{|l|}{ Weight -for-length $z$-score, mean \pm SD } \\
\hline \multicolumn{6}{|l|}{ Body system associated with the presenting symptoms } \\
\hline Cutaneous & $0.12 \pm 0.99$ & $-0.13 \pm 0.86$ & $0.07 \pm 0.88$ & $0.07 \pm 0.99$ & $0.43 \pm 0.94$ \\
\hline$p$-value vs. baseline & & 0.025 & 0.464 & 0.659 & 0.134 \\
\hline Gastrointestinal & $-0.88 \pm 1.23$ & $-0.38 \pm 1.25$ & $-0.39 \pm 1.31$ & $-0.09 \pm 1.36$ & $0.03 \pm 1.53$ \\
\hline$p$-value vs. baseline & & 0.003 & 0.004 & 0.000 & 0.009 \\
\hline Respiratory & $-0.24 \pm 1.32$ & $0.34 \pm 1.21$ & $0.20 \pm 1.68$ & $0.26 \pm 1.64$ & $-1.28 \pm 1.65$ \\
\hline$p$-value vs. baseline & & 0.194 & 0.216 & 0.122 & 0.075 \\
\hline \multicolumn{6}{|l|}{ Number of the presenting symptoms } \\
\hline 1 symptom & $-0.34 \pm 1.21$ & $-0.20 \pm 1.07$ & $-0.09 \pm 1.15$ & $0.01 \pm 1.21$ & $0.17 \pm 1.29$ \\
\hline$p$-value vs. baseline & & 0.150 & 0.045 & 0.007 & 0.002 \\
\hline$>1$ symptom & $-0.53 \pm 1.33$ & $-0.35 \pm 1.14$ & $-0.18 \pm 0.94$ & $-0.35 \pm 0.97$ & $-0.17 \pm 1.39$ \\
\hline$p$-value vs. baseline & & 0.601 & 0.378 & 0.414 & 0.187 \\
\hline
\end{tabular}

Abbreviation: SD: standard deviation

A $p$-value $<0.05$ indicates statistical significance (difference between diagnosis and $3^{\text {rd }}, 6^{\text {th }}, 9^{\text {th }}$, and $12^{\text {th }}$ month; paired Student's $t$-test).
A
Means of weight-for-age z-score
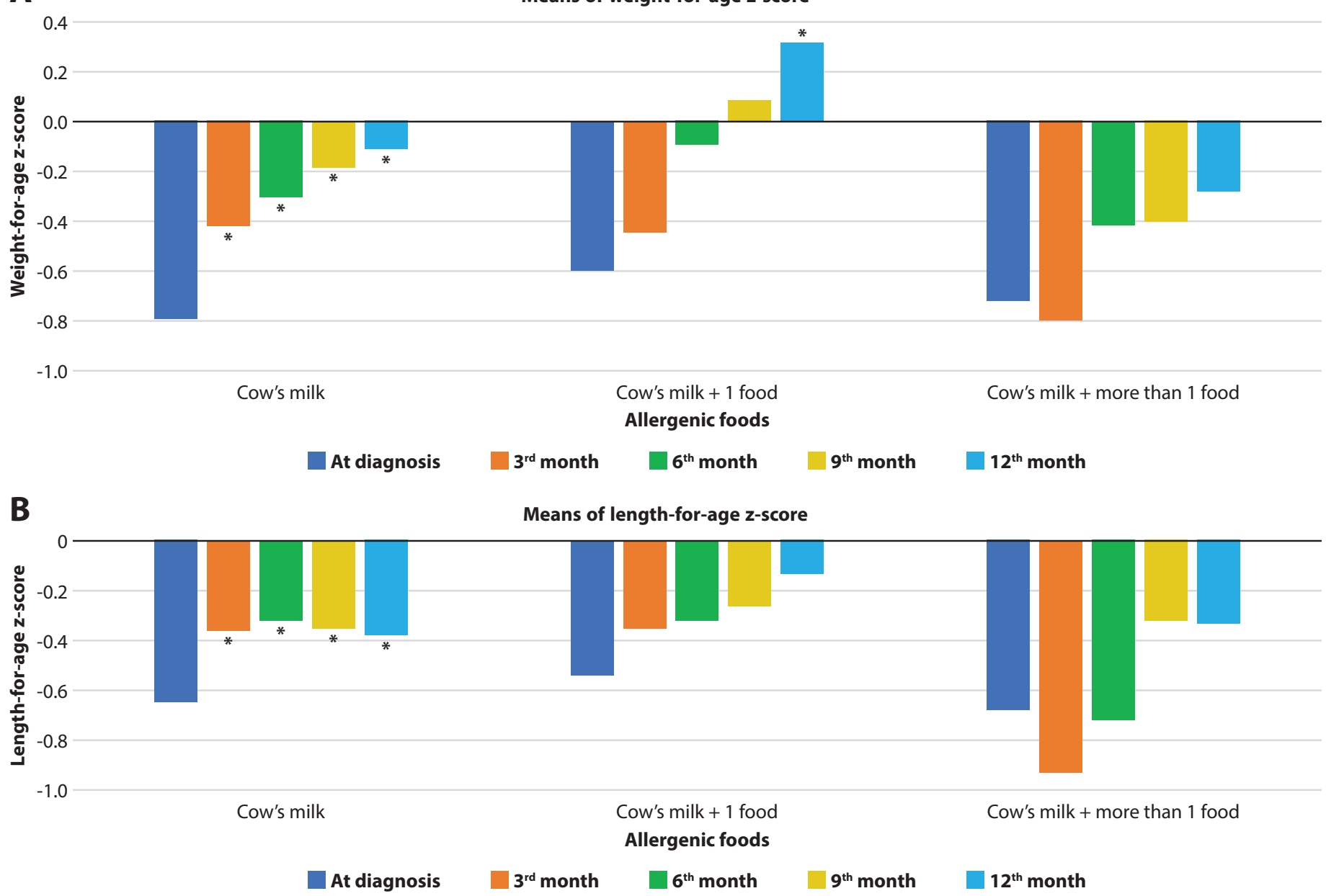

Figure 1. Infant growth parameters at diagnosis and at each follow-up time point according to the number of allergenic foods

${ }^{*}$ A $p$-value $<0.05$ indicates statistical significance (difference between diagnosis and $3^{\text {rd }}, 6^{\text {th }}, 9^{\text {th }}$, and $12^{\text {th }}$ month of each allergenic food group; paired Student's t-test). 


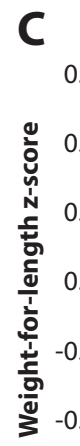

Means of weight-for-length z-score 0.6
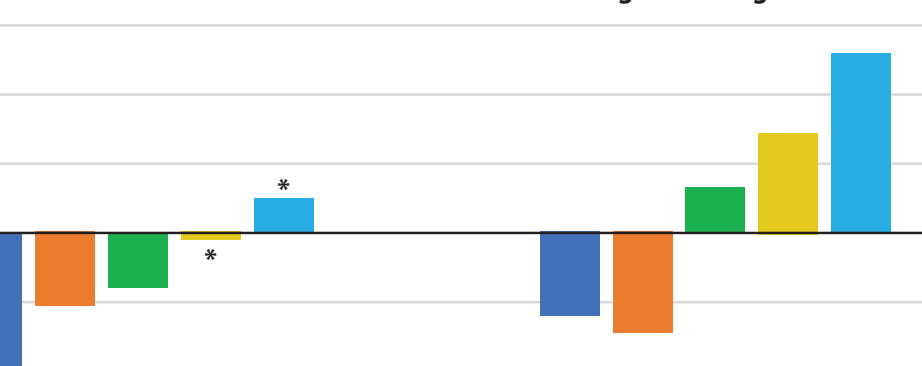

$-0.6$

Cow's milk

Cow's milk +1 food

Cow's milk + more than 1 food

Allergenic foods

At diagnosis $\square 3^{\text {rd }}$ month $\square 6^{\text {th }}$ month $\quad 9^{\text {th }}$ month $\quad 12^{\text {th }}$ month

Figure 1. (Continued)

Table 4. Univariate and multivariate analyses for factors independently associated with accession of tolerance to CMPA

\begin{tabular}{|c|c|c|c|c|c|c|}
\hline \multirow{3}{*}{ Factors } & \multicolumn{6}{|c|}{ Accession of tolerance to CMPA } \\
\hline & \multicolumn{3}{|c|}{ Univariate Cox regression analysis } & \multicolumn{3}{|c|}{ Multivariate Cox regression analysis } \\
\hline & Hazard ratio & $95 \% \mathrm{CI}$ & $p$-value & Hazard ratio & $95 \% \mathrm{CI}$ & $p$-value \\
\hline \multicolumn{7}{|l|}{ Age at diagnosis } \\
\hline$\geq 6$ months & 1 & & & - & - & - \\
\hline$<6$ months & 0.90 & $0.54-1.53$ & 0.721 & - & - & - \\
\hline
\end{tabular}

Presenting symptoms

\begin{tabular}{|c|c|c|c|c|c|c|}
\hline$>1$ symptom & 1 & & & 1 & & \\
\hline 1 symptom & 1.63 & $0.84-3.17$ & 0.147 & 2.82 & $0.79-10.04$ & 0.108 \\
\hline \multicolumn{7}{|c|}{ Serum cow's milk-specific IgE $(n=75)$} \\
\hline Positive & 1 & & & 1 & & \\
\hline Negative & 1.62 & $0.86-3.04$ & 0.132 & 1.12 & $0.48-2.65$ & 0.781 \\
\hline
\end{tabular}

Eosinophilia $(\mathrm{n}=84)$

\begin{tabular}{|c|c|c|c|c|c|c|}
\hline Positive & 1 & & & 1 & & \\
\hline Negative & 0.65 & $0.37-1.15$ & 0.145 & 0.73 & $0.34-1.55$ & 0.416 \\
\hline \multicolumn{7}{|l|}{ Alternative formulae or milk } \\
\hline Breast milk & 1 & & & 1 & & \\
\hline Soy formula & 0.29 & $0.11-0.73$ & 0.009 & 0.14 & $0.03-0.62$ & 0.009 \\
\hline EHF & 0.35 & $0.15-0.82$ & 0.016 & 0.21 & $0.07-0.64$ & 0.006 \\
\hline cAAF & 0.97 & $0.28-3.35$ & 0.969 & 0.39 & $0.07-2.20$ & 0.290 \\
\hline nAAF & 0.53 & 0.13-2.09 & 0.372 & 0.26 & $0.04-1.77$ & 0.172 \\
\hline Chicken-based formula & 0.65 & $0.20-2.11$ & 0.480 & 0.22 & $0.04-1.09$ & 0.064 \\
\hline \multicolumn{7}{|l|}{ Allergenic food(s) } \\
\hline Cow's milk + other food(s) & 1 & & & 1 & & \\
\hline Cow's milk & 2.09 & $1.11-3.94$ & 0.021 & 2.83 & $1.04-7.64$ & 0.040 \\
\hline
\end{tabular}

Abbreviations: CMPA: cow's milk protein allergy; CI: confidence interval; IgE: immunoglobulin E; EHF: extensively hydrolyzed protein formula; cAAF: commercial amino acid-based formula; nAAF: new amino acid-based formula

A $p$-value $<0.05$ indicates statistical significance. 
About $30 \%$ of infants (35 infants, of which $35 \%$ had IgE-mediated allergy and $65 \%$ had non-IgE-mediated allergy) tolerated cow's milk at one year of treatment. A larger percentage of these 35 infants were diagnosed with CMPA before 6 months of age ( $60 \%$ vs. $40 \%$ diagnosed after 6 months), and most of them were diagnosed before 9 months of age ( $83 \%$ vs. $17 \%$ diagnosed after 9 months).

The type of alternative formulae or milk and the number of allergenic foods were significantly associated with the development of tolerance to CMP in both univariate and multivariate Cox regression analyses (Table 4). Compared to breast milk, SF and EHF were both significantly associated with decreased probability of tolerance to CMP (HR: 0.14, 95\% CI: $0.03-0.62 ; p=0.009$, and HR: $0.21,95 \%$ CI: $0.07-0.64 ; p$ $=0.006$, respectively). Allergy to only CMP was significantly associated with increased probability of tolerance to CMP compared to allergy to CMP and other foods (HR: 2.83, 95\% CI: $1.04-7.64 ; p=0.040)$. There was no statistical difference in hazard ratio for development of tolerance to CMP relative to

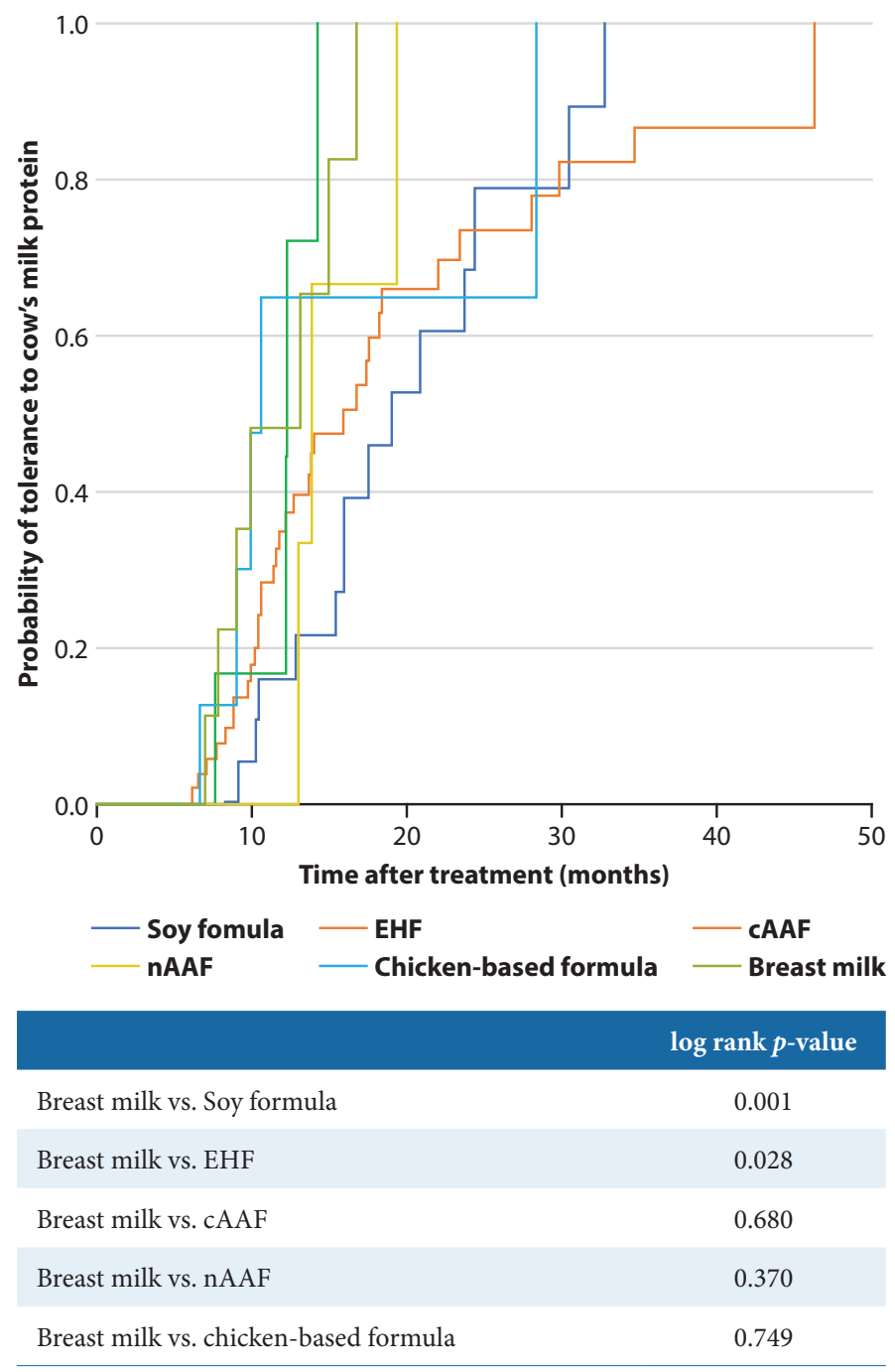

Figure 2. Kaplan-Meier curves of the estimated probability of tolerance to cow's milk protein in infants consuming five different alternative formulae and breast milk

Abbreviations: CI, confidence interval; EHF, extensively hydrolyzed protein formula; cAAF, commercial amino acid-based formula; nAAF, new amino acid-based formula age at diagnosis, presenting symptoms, presence or absence of serum cow's milk-specific IgE, or presence or absence of eosinophilia.

Kaplan-Meier survival analysis revealed the median time from treatment to the development of tolerance to CMP for the six feeding regimens to be 10.5 months for chicken-based formula, 12.2 months for cAAF, 13.1 months for breast milk, 13.8 months for nAAF, 15.9 months for EHF, and 19.0 months for SF (Figure 2). Log-rank test showed a significantly higher probability of tolerance to CMP in infants consuming breast milk compared to those receiving SF $(p=0.001)$ or $\operatorname{EHF}(p=$ $0.028)$.

\section{Discussion}

This study describes the growth defined according to WAZ, LAZ, and WLZ in infants newly diagnosed with CMPA. Marked improvement in growth, especially WAZ, was observed among those treated with EHF; in those who had gastrointestinal symptom; in those who presented with one symptom at diagnosis of CMPA; and in those who were allergic to only CMP. Number of infants with non-IgE-mediated CMPA tolerated CMP more than those with IgE-mediated CMPA after 1-year treatment. Tolerance was affected by early age of diagnosis. Compared to breast milk, SF and EHF were both found to be associated with decreased probability of tolerance to CMP. Moreover, infants with allergy to only CMP had a higher probability of developing tolerance to CMP than those allergic to CMP and to other foods.

At diagnosis of CMPA, the means of WAZ, LAZ, and WLZ among all infants were $-0.76,-0.64$, and -0.38 , respectively - all of which were within the normal ranges according to the World Health Organization (underweight defined as below -2 z-score weight-for-age, stunted defined as below $-2 \mathrm{z}$-score length/height-for-age, and wasting defined as below -2 z-score weight-for-length). ${ }^{20}$ Even though significantly increased weight was found only in the EHF and SF groups, infants consuming nAAF, cAAF, chicken-based formula, or breast milk still experienced some improvement in growth even though the difference from baseline was not statistically significant. The availability of alternative formula in Thailand has changed over time. Soy protein-based formula and EHCF have been available in Thailand for at least 30 years, whereas AAF and EHWF were more recently introduced within the last 10 years. This increase in the number of available alternatives may have influenced formula selection by physicians, and this would have influenced differences in the numbers of subjects in each group in this study. Consequently, most infants in the EHF group in this study (58/60) consumed EHCF. Furthermore, maternal diet during pregnancy and lactation affects the nutritional composition of breast milk..$^{21,22}$ Our study did not assess the nutritional aspects of breastfeeding mothers, such as the impact of elimination diet for CMP on infant growth. Infants who present with respiratory symptom might be dyspneic, which leads to decreased intake or withholding of oral food intake due to fear of aspiration, and this could cause no significant improvement in growth. Infants who present with more than one symptom might have a higher likelihood of decreased food intake, reduced nutrient absorption, and/or increased nutrient loss. ${ }^{2}$ 
Those with allergy to other foods that requires avoidance of those foods are at higher risk for impaired weight gain and linear growth. ${ }^{13,14}$

Several studies reported the effects of diet elimination for CMPA on children's growth. Children allergic to CMP and other foods have been reported to have significantly decreased relative weight, but unchanged relative height during a six-month follow-up that started at a mean age of two years (range: $0.6-4.1$ years) ${ }^{11}$ In that study, no substitute formula was prescribed to replace cow's milk for subjects older than one year. In addition, the mean age of subjects in that study was greater than the mean study age of subjects in our study. Both of those factors are different from our study, and both could influence unfavorable growth outcomes. In another study, children with CMPA-proven atopic dermatitis were reported to have decreased length SD score and weightfor-length index during diet elimination without catch-up by 24 months of age. Delayed growth was very noticeable in a subset of subjects with early onset compared to those with late onset symptom (mean age at onset of symptom: 2.7 vs. 6.5 months, and mean age at start of elimination diet: 4.6 vs. 12.5 months, respectively). ${ }^{10}$ The median age of elimination in our study was 4.1 months, which is consistent with the mean age of the early onset group in that study; however, our data shows growth improvement in overall subjects. Moreover, the two aforementioned studies did not perform subgroup analysis of growth among types of substitute formula, or relative to the presence or absence of other allergenic foods.

Several studies have described the effects of specified alternative formulae for CMPA on children's growth. Infants fed casein- or rice-based hydrolyzed formula showed a trend toward higher WAZ increments at 6 to 12 months of age, and both formulae effectuated greater improvement in weight-forage compared to soy formula. ${ }^{23}$ The growth of children with IgE-mediated CMPA treated with hydrolyzed rice protein formula was comparable to the growth of those receiving EHWF and EHCF, and their growth parameters up to 18 months of age were within normal range in spite of no significant changes over time. ${ }^{15}$ Infants with immediate and delayed reactions to CMP who were fed with EHCF showed improvement in WAZ and LAZ within a 4 -month study period. ${ }^{16}$ The effect on the growth of healthy term infants fed with a new amino acid-based formula was comparable to that observed among those fed with EHCF during a 120-day study period. ${ }^{24}$ Infants not responding to EHF had significant increase in weight z-score change after switching to an amino acid-based formula for 12 weeks, as well as improvement in allergic symptoms. ${ }^{18}$ These studies show improvement in weight and length after treatment with specified alternative formulae for at least 12 weeks to 18 months, which is consistent with our data.

Appropriate nutritional intervention in malnourished children results in growth improvement, but weight catch-up was more rapid than length catch-up. ${ }^{25}$ This supports our findings that weight gain was found earlier than linear growth after treatment with alternative formulae.

The underlying mechanisms in accession of tolerance to CMP remain obscure. One of the predictive factors of clinical tolerance is a continuing reduction in sIgE. In contrast, higher sIgE levels to epitopes of $\beta$-casein and $\alpha(\mathrm{s} 1)$-casein have been identified to associate with persistence of CMPA. ${ }^{26}$ These may explain that clinical tolerance is reached in infants with non-IgE-mediated CMPA more than those with IgE-mediated CMPA. Early diagnosis of CMPA and treating with elimination diet enhance a reduction in sIgE levels, which may correlate with the accession of tolerance.

Multivariate Cox regression analysis showed that none of the evaluated feeding regimens had a significant hazard ratio relative to the development of tolerance to CMP compared to breast milk. This is probably due to the immunoregulatory properties of transforming growth factor- $\beta$ on decreasing allergenic response, as shown in animal studies. ${ }^{27-29}$ Infants on breast milk of this study were diagnosed as CMPA at a median age of 2.6 months, and their major diagnosis (50\% of subjects) was food protein-induced allergic proctocolitis (FPIAP), which is consistent with a previous study that found FPIAP in as many as $60 \%$ of breastfed infants. ${ }^{30}$ Moreover, according to the natural history of FPIAP, tolerance to allergenic food usually occurs by the age of one year. ${ }^{30,31}$ This may explain the early tolerance to CMP in the breast milk group.

The clinical implications of our findings relative to the treatment of CMPA include: (a) For breastfed infants, provision of breastfeeding with maternal elimination for CMP, maternal dietary counseling to prevent nutritional deficiencies, and prescribing calcium supplements should be emphasized; (b) For formula-fed infants, commercial alternative formulae, if available and affordable, are recommended; otherwise, clinically-proven innovative formulae may be an appropriate alternative; and, (c) During a period of complementary feeding, timing of introduction, food choices, additional food allergies, and alternative sources for nutrients should also be considered in order to achieve normal nutritional status and growth outcome.

This study has some limitations. First, its retrospective design made it vulnerable to incomplete information and a different number of subjects among alternative formulae or milk groups. Second, we enrolled infants from only one center. Third, diagnosis of CMPA was not confirmed by a double-blind placebo-controlled food challenge, which is the gold standard. Fourth, the difference in growth pattern seen among alternative formulae might be due to difference in clinical severity/pre-existing malnutrition e.g. there is a trend towards lower baseline WAZ/LAZ in both AAF groups but the sample size is too small to show significant difference. Fifth, factors related to food choice (e.g. availability, awareness, and cost) were not explored. And last, feeding advice may vary among pediatricians, and these variations were not accounted for in this study. However, the establishment of the clinical practice guideline for diagnosis and management of cow milk protein allergy for Thais in 2012 might reduce variation in advice for CMP avoidance. ${ }^{32}$ The strengths of this study include the inclusion of infants consuming breast milk only while their mothers were practicing CMP avoidance; the inclusion of two new alternative formulae; and, our investigation of the effects of allergenic foods other than CMP.

In conclusion, twelve-month growth improvement, especially in weight, was observed to be more pronounced in CMPA infants fed with EHF, while other feeding regimen groups resulted in no significant change in growth, but still 
were within the normal range. Infants who had gastrointestinal symptoms, one symptom at diagnosis, or allergy to only CMP without allergy to other foods had better weight gain. Probability of accession of tolerance to CMP was found to be associated with breast milk as the therapeutic dietary choice. Future prospective randomized controlled multicenter studies including long-term growth, time to the development of tolerance, specific food intake of infants and mothers, dietary choices, and laboratory tests to assess nutritional status are warranted.

\section{Acknowledgements}

All authors declare no conflicts of interest, and no financial support from the companies that produce the medical formulas described in this report. The authors gratefully acknowledge Suranee Chalermchainukul, Supawan Kunnangja, and Hathaichanok Rukprayoon for research coordination; Julaporn Pooliam for statistical analysis assistance; and Dr. Ruangvith Tantibhaedhyangkul and Asst. Prof. Kevin P. Jones for proofreading a draft of this manuscript. C.T. was responsible for data collection, data analysis and interpretation, and preparation of the draft manuscript, and N.D. was responsible for study conception and design, data collection, data analysis and interpretation, preparation of the draft manuscript, and critical revision of the manuscript. Both authors have read and approved the final version of the manuscript.

\section{References}

1. Høst A. Frequency of cow's milk allergy in childhood. Ann Allergy Asthma Immunol. 2002;89:33-7.

2. Lifschitz C, Szajewska H. Cow's milk allergy: evidence-based diagnosis and management for the practitioner. Eur J Pediatr. 2015;174:141-50.

3. American Academy of Pediatrics. Committee on Nutrition. Hypoallergenic infant formulas. Pediatrics. 2000;106:346-9.

4. Zeiger R, Sampson H, Bock SA, Burks AW, Harden K, Noone S, et al. Soy allergy in infants and children with IgE mediated cow's milk allergy. J Pediatr. 1999;134:614-22.

5. Koletzko S, Niggemann B, Arato A, Dias JA, Heuschkel R, Husby S, et al. Diagnostic approach and management of cow's-milk protein allergy in infants and children: ESPGHAN GI Committee Practical Guidelines. J Pediatr Gastroenterol Nutr. 2012;55:221-9.

6. Jirapinyo P, Densupsoontorn N, Wongarn R, Thamonsiri N. Comparisons of a chicken-based formula with soy-based formula in infants with cow milk allergy. Asia Pac J Clin Nutr. 2007;16:711-5.

7. Jirapinyo P, Densupsoontorn N, Kangwanpornsiri C, Wongarn R. Chicken-based formula is better tolerated than extensively hydrolyzed casein formula for the management of cow milk protein allergy in infants. Asia Pac J Clin Nutr. 2012;21:209-14.

8. Jirapinyo P, Densupsoontorn N, Kangwanpornsiri C, Wongarn R, Tirapongporn $\mathrm{H}$, Chotipanang $\mathrm{K}$, et al. Improved tolerance to a new amino acid-nased formula by infants with cow's milk protein allergy. Nutr Clin Pract. 2016;31:824-8.

9. Jirapinyo P, Densupsoontorn N, Kangwanpornsiri C, Wongarn R, Tirapongporn $\mathrm{H}$, Chotipanang $\mathrm{K}$, et al. Reconfirmation of improved tolerance to a new amino acid-based formula by infants with cow's milk protein allergy. Asia Pac J Clin Nutr. 2017;26:1088-91.

10. Isolauri E, Sutas Y, Salo MK, Isosomppi R, Kaila M. Elimination diet in cow's milk allergy: risk for impaired growth in young children. J Pediatr. 1998;132:1004-9.

11. Paganus A, Juntunen-Backman K, Savilahti E. Follow-up of nutritional status and dietary survey in children with cow's milk allergy. Acta Paediatrica. 1992;81:518-21.

12. Tiainen JM, Nuutinen OM, Kalavainen MP. Diet and nutritional status in children with cow's milk allergy. Eur J Clin Nutr. 1995;49:605-12.

13. Christie L, Hine RJ, Parker JG, Burks W. Food allergies in children affect nutrient intake and growth. J Am Diet Assoc. 2002;102:1648-51.
14. Sova C, Feuling MB, Baumler M, Gleason L, Tam JS, Zafra H, et al. Systematic review of nutrient intake and growth in children with multiple IgE-mediated food allergies. Nutr Clin Pract. 2013;28:669-75.

15. Reche M, Pascual C, Fiandor A, Polanco I, Rivero-Urgell M, Chifre R, et al. The effect of a partially hydrolysed formula based on rice protein in the treatment of infants with cow's milk protein allergy. Pediatr Allergy Immunol. 2010;21:577-85.

16. Dupont C, Bradatan E, Soulaines P, Nocerino R, Berni-Canani R. Tolerance and growth in children with cow's milk allergy fed a thickened extensively hydrolyzed casein-based formula. BMC Pediatrics. 2016;16:96.

17. Kangwanpornsiri C, Jirapinyo P, Densupsoontorn N, Poungkaew J, Wongarn R, Thammonsiri N, et al. Growth of infants with cow's milk allergy fed chicken-based formula. J Med Assoc Thai. 2013;96:20-5.

18. Vanderhoof J, Moore N, de Boissieu D. Evaluation of an amino acid-based formula in infants not responding to extensively hydrolyzed protein formula. J Pediatr Gastroenterol Nutr. 2016;63:531-3.

19. WHO Anthro for personal computers, version 3.2.2, 2011: Software for assessing growth and development of the world's children [software]. Geneva: WHO; 2010 [cited 2019 Dec 10]. Available from: http://www.who. int/childgrowth/software/en/.

20. World Health Organization [Internet]. Geneva: WHO; c2008-20 [cited 2019 Dec 7]. Training course on child growth assessment; [about 58 p.]. Available from: https://www.who.int/childgrowth/training/module_c_ interpreting_indicators.pdf

21. Barrera C, Valenzuela R, Chamorro R, Bascuñán K, Sandoval J, Sabag N, et al. The impact of maternal diet during pregnancy and lactation on the fatty acid composition of erythrocytes and breast milk of chilean women. Nutrients. 2018;10:839.

22. Amezcua López JA, Solís Pacheco JR, García Morales E, Gutiérrez Padilla JA, Zepeda Morales ASM, Angulo Castellanose E, et al. Influence of the diet of Mexican women on the nutritional quality and the presence of beneficial microorganisms in human milk. Nutr Hosp. 2019;36:1139-49.

23. Agostoni C, Fiocchi A, Riva E, Terracciano L, Sarratud T, Martelli A, et al. Growth of infants with IgE-mediated cow's milk allergy fed different formulas in the complementary feeding period. Pediatr Allergy Immunol. 2007;18:599-606

24. Burks W, Jones SM, Berseth CL, Harris C, Sampson HA, Scalabrin DM. Hypoallergenicity and effects on growth and tolerance of a new amino acid-based formula with docosahexaenoic acid and arachidonic acid. J Pediatr. 2008;153:266-71.

25. Kleinman RE, Greer FR, editors. American Academy of Pediatrics Committee on Nutrition. Failure to thrive. In: Kleinman RE, Greer FR, editors. Pediatric Nutrition. 7th ed. Elk Grove Village, IL: American Academy of Pediatrics; 2014: p. 663-700.

26. Martorell-Aragonés A, Echeverría-Zudaire L, Alonso-Lebrero E, Boné -Calvo J, Martín-Muñoz MF, Nevot-Falcó S, et al. Position document: IgE-mediated cow's milk allergy. Allergol Immunopathol (Madr). 2015; 43:507-26.

27. Oddy WH, Rosales F. A systematic review of the importance of milk TGF-beta on immunological outcomes in the infant and young child. Pediatr Allergy Immunol. 2010;21:47-59.

28. Nakao A. The role and potential use of oral transforming growth factor- $\hat{\mathrm{I}}^{2}$ in the prevention of infant allergy. Clin Exp Allergy. 2010;40:725-30.

29. Holvoet S, Perrot M, de Groot N, Prioult G, Mikogami T, Verhasselt V, et al. Oral tolerance induction to newly introduced allergen is favored by a transforming growth factor-beta-enriched formula. Nutrients. 2019;11: 2210.

30. Maloney J, Nowak-Wegrzyn A. Educational clinical case series for pediatric allergy and immunology: allergic proctocolitis, food protein -induced enterocolitis syndrome and allergic eosinophilic gastroenteritis with protein-losing gastroenteropathy as manifestations of non- $\operatorname{IgE}$ -mediated cow's milk allergy. Pediatr Allergy Immunol. 2007;18:360-7.

31. Meyer R, Chebar Lozinsky A, Fleischer DM, Vieira MC, Toit GD, Vandenplas Y, et al. Diagnosis and management of Non-IgE gastrointestinal allergies in breastfed infants-An EAACI Position Paper. Allergy. 2020;75: 14-32.

32. Vivatvekin B, Suthutvoravut U, Benjaponpitak S, The Royal College of Pediatricians of Thailand, Thai Society of Pediatric Gastroenterology and Hepatology, Society of Pediatric Nutrition of Thailand, et al. Clinical practice guideline for diagnosis and management of cow milk protein allergy. Bangkok: Medi Journal Co. Ltd.; 2012. 